\title{
Previsão de desempenho de alunos baseado em construtos de autorregulação da aprendizagem
}

\author{
Rodrigo Lins Rodrigues ${ }^{1}$, Jorge L. C. Ramos ${ }^{3}$, João C. Sedraz Silva ${ }^{3}$, Thiago S. Araújo ${ }^{2}$, \\ Hugo V. L. Souza ${ }^{2}$, Fernando da F. de Souza ${ }^{2}$, Erik de G. Zambom ${ }^{3}$, Alex S. Gomes ${ }^{2}$ \\ ${ }^{1}$ Universidade Federal Rural de Pernambuco \\ Recife - PE - Brasil \\ ${ }^{2}$ Universidade Federal de Pernambuco \\ Recife-PE, Brasil \\ ${ }^{3}$ Universidade Federal do Vale do São Francisco \\ Juazeiro - BA - Brasil.
}

rodrigo.linsrodrigues@ufrpe.br, \{jorge.cavalcanti,joao.sedraz\}@univasf.ed
u.br, thiagosoara@gmail.com, \{hvls, fdfd, egz, asg\}@cin.ufpe.br

\begin{abstract}
The present article investigates the applicability of the Logistic Regression model to the prediction of the performance of students, belonging to $E A D$ courses, from the variables of the OSLQ collection instrument. A survey was conducted with 408 participants of courses in the EAD modality. Data collection was performed through a hybrid approach between questionnaire and academic performance variables extracted from the Moodle platform, in order to verify if the instrument was sufficient to predict the performance of students with satisfactory rates of accuracy. As a result, an accuracy value of $85.3 \%$ correct prediction, 84.8 accuracy and 92\% ROC curve was obtained. These results demonstrated that there is a possible relationship to be modeled between self-regulating learning abilities and students' academic performance.
\end{abstract}

Resumo. O presente artigo investiga a aplicabilidade do modelo de Regressão Logística para a previsão de desempenho de alunos, pertencentes de cursos de EAD, a partir das variáveis do instrumento de coleta OSLQ. Foi realizada uma pesquisa com 408 participantes de cursos na modalidade EAD. A coleta dos dados foi realizada através de uma abordagem híbrida entre questionário e variáveis de desempenho acadêmico extraídas da plataforma Moodle, com o objetivo de verificar se o instrumento era suficiente para prever o desempenho de alunos com taxas satisfatórias de acurácia. Como resultado, foi obtido um valor de acurácia de $85,3 \%$ de previsão correta, 84,8 de precisão e $92 \%$ de curva ROC. Estes resultados demonstraram que existe uma relação possível de ser modelada entre as habilidades de autorregulação da aprendizagem com o desempenho acadêmico dos alunos. 
VI Congresso Brasileiro de Informática na Educação (CBIE 2017)

Anais do XXVIII Simpósio Brasileiro de Informática na Educação (SBIE 2017)

\section{Introdução}

A autorregulação da aprendizagem é uma habilidade cognitiva por meio da qual pessoas conseguem gerir recursos, tempo, esforço, ambiente, interação e a busca por ajuda no processo de aprendizagem (Chen 2002); (Pintrich 1999); (Zimmerman e Martinez-Pons 1988). Estudos vêm sendo desenvolvidos para analisar a importância de habilidades da autorregulação no aprendizado em ambientes de aprendizagem online.

Estudantes que detém destas habilidades conseguem controlar o ritmo, o direcionamento do processo de aprendizagem e gerenciar um conjunto de características do ambiente de aprendizagem, realizando escolhas que o auxiliam no desenvolvimento de suas funções cognitivas (Broadbent e Poon 2015) .

Pesquisadores como Dabbagh e Kitsantas (2005) e Schunk e Zimmerman (1998) acreditam que os ambientes de aprendizagem on-line exigem do aluno uma maior consciência de seu processo de aprendizagem. Estes autores acreditam que estas habilidades são essenciais para o sucesso da aprendizagem autônomas (autodirigidas) e que o desenvolvimento dessas habilidades pode ser mensurado e apresentam relações com o desempenho acadêmico dos estudantes (Azevedo 2005); (Dabbagh e Kitsantas 2004).

Como forma de mensurar estas habilidades, diversos instrumentos de mensuração da autorregulação da aprendizagem vem sendo desenvolvidas ao longo dos anos, Ainda existem poucos instrumentos de análise específicos para o contexto da educação online. Um instrumento que vêm sendo utilizado na literatura é o questionário desenvolvido no artigo de Barnard, Lan, et al., (2009). Este instrumento é intitulado OSLQ - Online Selfregulated Learning Questionnaire. Foi desenvolvido e projetado para a avaliação de habilidades de autorregulação que são importantes na aprendizagem on-line.

Diante deste contexto, este trabalho tem como objetivo a aplicabilidade do instrumento OSLQ, e seus respectivos construtos existentes na escala, para realizar a previsão do desempenho de alunos em cursos à distância baseado em suas habilidades de autorregulação.

\section{Autorregulação da aprendizagem em ambientes de EAD}

Estudos vêm sendo desenvolvidos com objetivo de analisar a importância de habilidades da autorregulação da aprendizagem em ambientes de EAD. Diversos pesquisadores acreditam que os ambientes de aprendizagem on-line exigem do aluno uma maior responsabilidade para o processo de aprendizagem (Dabbagh \& Kitsantas, 2005); (Schunk \& Zimmerman, 1998).

Estas habilidades podem ser importantes para alunos que participam de cursos nesta modalidade (Dabbagh \& Kitsantas, 2004); (Garrison, 2003); (Hartley \& Bendixen, 2001); (Schunk \& Zimmerman, 1998). Por exemplo, Dabbagh e Kitsantas (2004) argumentaram que, "em um ambiente de aprendizagem baseado na Web, os alunos devem exercer um alto grau de competência de autorregulação para realizar seus objetivos de aprendizagem, enquanto que em ambientes tradicionais face-a-face em sala de aula, o instrutor exerce um controle significativo sobre o processo de aprendizagem e é capaz de monitorar a atenção do aluno e do seu progresso". Da mesma forma, autores como Kinzie (1990) afirmam que a habilidade de autorregulação é um dos três requisitos críticos para o sucesso do aluno. 
VI Congresso Brasileiro de Informática na Educação (CBIE 2017)

Anais do XXVIII Simpósio Brasileiro de Informática na Educação (SBIE 2017)

A utilização eficaz das estratégias de SRL é essencial em situações de aprendizagem flexíveis, devido ao alto grau de autonomia do aluno, resultante da ausência física do professor.

\subsection{Relações com o desempenho acadêmico}

O desempenho acadêmico pode ser definido como o ato de alcançar um resultado esperado em uma atribuição, atividade, prova ou grau de entendimento sobre um determinado assunto, onde é normalmente expresso em termos de pontuação numérica (Richardson, Abraham, \& Bond, 2012). Diversas pesquisas vem revelando a relação positiva entre o uso de estratégias SRL e os resultados acadêmicos em contextos de aprendizagem presencial (Beishuizen \& Steffens, 2011); (Dignath \& Buttner, 2008); (Pintrich, 2004); (Richardson, Abraham, \& Bond, 2012); (Zimmerman, 2008); (Wang, Doll, Deng, Park, \& Yang, 2013); (Agustiani, Cahyad, \& Musa, 2016). Estas pesquisas dão ênfase em ações de metacognição, gestão do tempo, e regulação de esforço (Richardson, Abraham, \& Bond, 2012).

No entanto, ainda são poucas as pesquisas conduzidas sobre o uso de SRL na aprendizagem on-line e suas relações com desempenho acadêmico (Broadbent \& Poon, 2015). A maioria dos trabalhos são estudos prospectivos (Carson, 2011); (ChanLin, 2012); (Cho \& Shen, 2013); (Hodges \& Kim, 2010); (Johnson, Gueutal, \& Falbe, 2009); (Michinov, Brunot, Le Bohec, Juhel, \& Delaval, 2011), seguidos por estudos experimentais (Chang, 2010); (Van den Boom, Paas, \& van Merriënboer, 2007) e transversais (Klingsieck, Fries, Horz, \& Hofer, 2012); (Wang, Shannon, \& Ross, 2013).

Em relação aos estudos prospectivos, o trabalho de Carson (2011) analisou o grau de sucesso de estudantes aplicando a escala de aprendizagem online LASSI (LLO). O autor fornece evidências de previsibilidade de desempenho, baseado nos itens da escala LASSI com uma acurácia global de $67 \%$.

No trabalho de Cho e Shen (2013) o autor discute a importância da orientação para as metas individuais dos estudantes e a relação com seu desempenho acadêmico. Os resultados mostraram uma relação positiva entre a quantidade de tempo que o aluno passa na plataforma, gestão do tempo, e seu desempenho acadêmico.

Em relação aos estudos transversais, um dos trabalhos que merece destaque é o trabalho de (Klingsieck, Fries, Horz, \& Hofer, 2012) o estudo dá ênfase ao problema de procrastinação dos estudantes e suas implicações no desempenho de atividades acadêmicas. Embora existam pesquisas sobre a procrastinação de estudantes, é pouco comum estudos destinados a entender a procrastinação em ambientes de ensino a distância.

\section{Percurso Metodológico}

Neste trabalho foi utilizada a técnica de regressão logística para a construção de um modelo de previsão a partir dos dados de questionário e dados de desempenho acadêmico de alunos extraídos da plataforma Moodle.

\subsection{Instrumento de coleta}

O instrumento de coleta utilizado nesta pesquisa foi adaptado do questionário de autorregulação da aprendizagem on-line (OSLQ) desenvolvido por (Barnard, Lan e 
VI Congresso Brasileiro de Informática na Educação (CBIE 2017)

Anais do XXVIII Simpósio Brasileiro de Informática na Educação (SBIE 2017)

Crooks, et al. 2008) e (Barnard, Lan e To, et al. 2009). Este instrumento é uma escala de 24 itens com um formato de resposta do tipo escala de Likert de 5 pontos com valores variando de 'concordo totalmente' (5) até ‘discordo totalmente' (1) (Tabela 1).

As variáveis são agrupadas nas seguintes categorias: Estabelecimento de Metas (EM), Estruturação do Ambiente (EA), Estratégias para realização de Tarefas (ET), Gerenciamento do Tempo (GT), Busca Por Ajuda (BA) e Auto Avaliação (AA).

Tabela 1. Variáveis do questionário de autorregulação da aprendizagem.

\begin{tabular}{|c|c|c|}
\hline $\mathbf{N}$ & Item do questionário & Construtos \\
\hline 1 & Eu defino metas para a realização das minhas tarefas em cursos on-line. & \multirow{5}{*}{$\begin{array}{l}\text { Estabelecimento } \\
\text { de metas (EM) }\end{array}$} \\
\hline 2 & Eu defino metas de curto prazo (diário ou semanal), bem como metas de longo prazo. & \\
\hline 3 & Eu mantenho um alto padrão de aprendizagem no meu curso. & \\
\hline 4 & Eu defino metas que me ajudam com o tempo de estudo dedicado para os meus cursos à distância. & \\
\hline 5 & Eu não comprometo a qualidade do meu trabalho porque é em EAD. & \\
\hline 6 & Eu escolho o local onde eu estudo para evitar distrações. & \multirow{4}{*}{$\begin{array}{l}\text { Estruturação do } \\
\text { Ambiente (EA) }\end{array}$} \\
\hline 7 & Eu procuro um lugar confortável para estudar. & \\
\hline 8 & Eu sei onde posso estudar de forma mais eficiente quando me dedico a EAD. & \\
\hline 9 & Eu escolho um horário do dia para estudar que tenha poucas distrações. & \\
\hline 10 & Eu tento fazer esquemas e anotações relacionadas com os conteúdos. & \multirow{4}{*}{$\begin{array}{l}\text { Estratégia para } \\
\text { realização de } \\
\text { tarefas (ET) }\end{array}$} \\
\hline 11 & Eu costumo ler os materiais em voz alta para não sofrer distrações. & \\
\hline 12 & Eu preparo minhas perguntas antes de entrar nos chats e fóruns de discussões. & \\
\hline 13 & Costumo utilizar material extra do que foi disponibilizado na plataforma. & \\
\hline 14 & Eu reservo tempo extra para estudar para minhas disciplinas da EAD. & \multirow{3}{*}{$\begin{array}{l}\text { Gerenciamento } \\
\text { do Tempo (GT) }\end{array}$} \\
\hline 15 & Agendo dias específicos da semana para estudar para meu curso & \\
\hline 16 & Tento distribuir meu tempo estudando uniformemente todos os dias. & \\
\hline 17 & Procuro amigos para tirar dúvidas sobre os conteúdos quando preciso. & \multirow{4}{*}{$\begin{array}{l}\text { Busca por ajuda } \\
\text { (BA) }\end{array}$} \\
\hline 18 & Compartilho meus problemas com os meus colegas descobrir como resolver os nossos problemas. & \\
\hline 19 & Quando necessário, eu tento encontrar meus colegas de curso presencialmente. & \\
\hline 20 & Eu costumo pedir ajuda ao tutor ou professor através de e-mail ou mensagem via plataforma. & \\
\hline 21 & Eu costumo fazer um resumo do meu aprendizado para refletir sobre o que aprendi na disciplina. & \multirow{4}{*}{$\begin{array}{c}\text { Auto } \\
\text { avaliação (AA) }\end{array}$} \\
\hline 22 & Faço reflexão e questionamentos sobre o material do curso disponibilizado. & \\
\hline 23 & Me comunico com meus colegas para refletir como está meu andamento no curso. & \\
\hline 24 & Costumo fazer comparativos entre o que estou aprendendo e o que meus colegas estão aprendendo. & \\
\hline
\end{tabular}

Por meio da aplicação do questionário foi coletada uma amostra de alunos da Universidade <omitido para revisão> que promove, a mais de dez anos, cursos de graduação na modalidade de EAD através do programa Universidade Aberta do Brasil.

Os dados foram coletados por meio de um processo de amostragem aleatória simples (Bolfarine \& Bussab, 2005), totalizando uma amostra de 408 alunos, divididos em quatro cursos: Letras, Administração, Biologia e Pedagogia, com idade média de 30 $(\mathrm{SD}=18,23)$ anos. A figura 1 apresenta o processo de coleta, análise e validação.

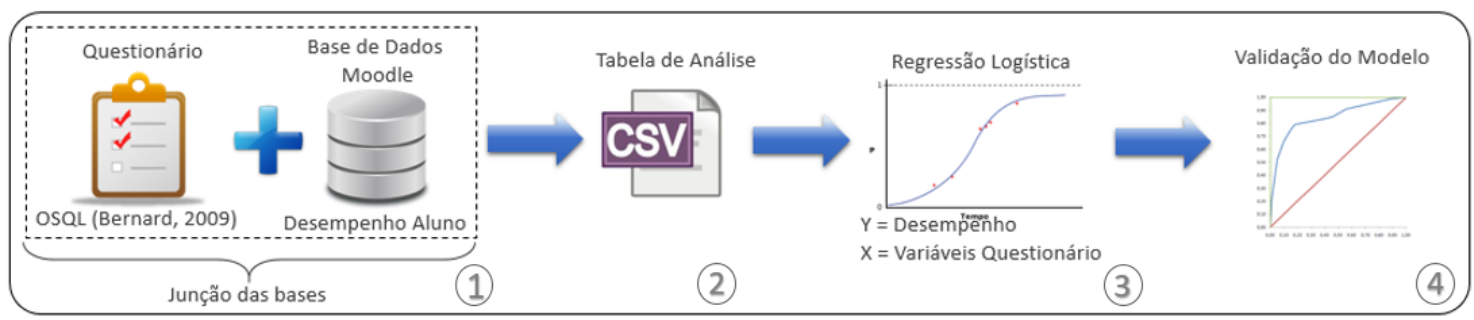

Figura 1. Processo de análise e construção do modelo logístico

Após a aplicação do questionário, foram identificadas e extraídas as variáveis, registrados no banco de dados da plataforma Moodle, relacionadas ao desempenho acadêmico de cada um dos alunos respondentes do questionário. 
De posse de ambas as bases de dados, a primeira fase foi a realização de uma integração (1), através da chave primária de ambas as bases de dados (questionário OSLQ + Desempenho dos alunos) com o objetivo de construir uma base de análise contendo as respostas dos alunos e seus respectivos desempenhos acadêmicos registrados na plataforma Moodle. A segunda fase (2) foi a geração de uma só base que contivesse dados do questionário e os desempenhos dos alunos.

A terceira fase (3) teve como objetivo a construção de um modelo inferencial capaz de prever o desempenho do aluno, baseado nas suas afirmações relacionadas à habilidades de autorregulação da aprendizagem. Para o processo de modelagem foi realizado por meio da linguagem $\mathrm{R}^{1}$ e utilizado a técnica de Análise de Regressão Logística.

A última fase foi a realização da validação do modelo (4) construído através das métricas de: Acurácia, Precisão e Curva ROC. Para a plotagem do gráfico da curva ROC e o cálculo de área, foram utilizados os pacotes $R O C R^{2}$, plotROC $^{3}$, ggplot $^{4}$ da plataforma R. Na seção seguinte são apresentados os resultados descritivos, a modelagem e as respectivas métricas de validação.

\section{Resultados e Discussões}

Os resultados foram divididos em duas fases, a primeira fase mostra estatísticas exploratórias sobre o público alvo e as variáveis do questionário. A segunda fase apresenta o modelo de previsão de desempenho e suas respectivas métricas de avaliação.

\subsection{Análise exploratória}

Inicialmente foi construída uma tabela de frequência com a especificação do curso ao qual o aluno estava vinculado. Os cursos que oferecidos pela instituição são três licenciaturas e um bacharelado. A Tabela 2 mostra a frequência do quantitativo de alunos por curso.

Tabela 2. Variáveis de interação coletadas.

\begin{tabular}{l|cccc}
\hline Cursos & Frequência & Freq. Rel. & Freq. Perc. & Freq. Acum. \\
\hline Administração Pública & 27 & 0,07 & 6,62 & 6,62 \\
Licenciatura em Biologia & 112 & 0,27 & 27,45 & 34,07 \\
Licenciatura em Letras & 88 & 0,22 & 21,57 & 55,64 \\
Licenciatura em Pedagogia & 170 & 0,42 & 41,67 & 97,30 \\
Não Informado & 11 & 0,00 & 2,70 & 100,00 \\
$\quad$ Total & $\mathbf{4 0 8}$ & & & \\
\hline
\end{tabular}

Dos alunos que fizeram parte da amostra $57,7 \%$ possuem apenas ensino médio, $24,22 \%$ já cursaram algum curso superior, seja completo ou parcialmente e $7,35 \%$ possuem pós-graduação, nos níveis especialização, mestrado ou doutorado. A figura 24 mostra o gráfico com os resultados da predominância de cada um dos construtos para a amostra selecionada.

\footnotetext{
${ }^{1}$ https://cran.r-project.org/

${ }^{2}$ https://cran.r-project.org/web/packages/ROCR/ROCR.pdf

${ }^{3} \mathrm{https}$ ://cran.r-project.org/web/packages/plotROC/plotROC.pdf

${ }^{4}$ https://cran.r-project.org/web/packages/ggplot2/ggplot2.pdf
} 
VI Congresso Brasileiro de Informática na Educação (CBIE 2017)

Anais do XXVIII Simpósio Brasileiro de Informática na Educação (SBIE 2017)

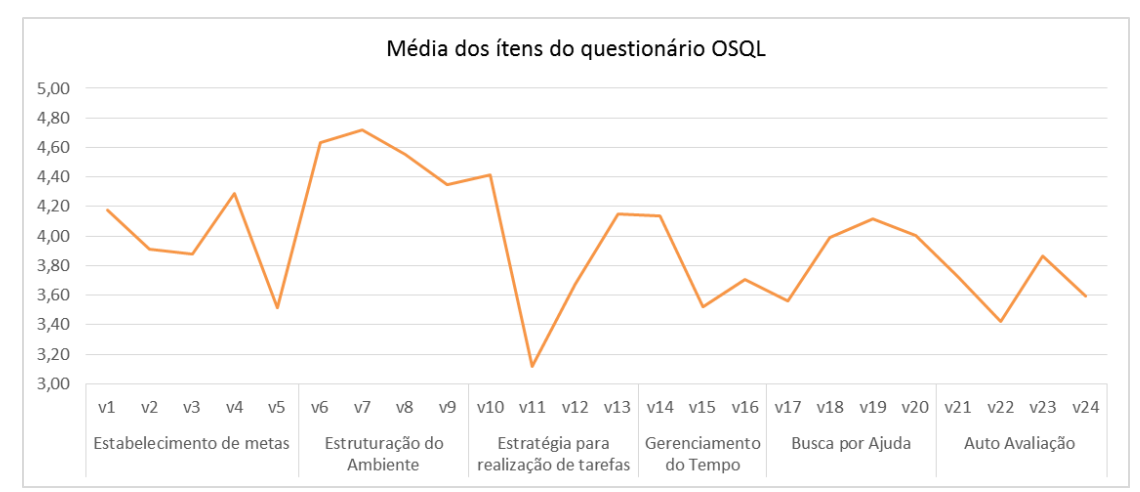

Figura 2. Gráfico das médias de respostas por construto de autorregulação.

De acordo com o gráfico, podemos observar que o construto que obteve maiores valores médios foi o construto de Estruturação do Ambiente, dos quais a variável V7 (Eu procuro um lugar confortável para estudar) obteve o maior resultado médio. Isto representa uma predominância na habilidade dos alunos na organização do seu ambiente de estudo.

Os construtos que apresentaram menores médias, em relação a afirmação dos alunos, foi o construto relacionado a Gestão do Tempo e o construto de Auto Avaliação. Isto nos mostra que, dentre todas as habilidades de autorregulação da aprendizagem, a habilidade do aluno em organizar e gerir seu tempo de estudo é uma das mais deficientes, seguida pela baixa habilidade de auto avaliar seu processo de aprendizagem.

Os resultados desta fase serviram para ter uma visão inicial e exploratória sobre o comportamento autorregulatório dos alunos da instituição, a qual a pesquisa foi realizada. A próxima seção descreve a modelagem realizada a partir destes dados para o desenvolvimento de um modelo de previsão de desempenho dos alunos.

\subsection{Modelagem através da regressão logística}

Por meio do modelo de regressão logística foi possível entender o comportamento da variável dependente binária (Desempenho) com base em um conjunto de variáveis independentes numéricas ou categóricas (variáveis do questionário de autorregulação da aprendizagem):

$$
\operatorname{logit}\left(p_{i}\right)=\ln \left(\frac{p_{i}}{1-p_{i}}\right)=\beta_{0}+\beta_{1} x_{1}+\cdots+\beta_{n} x_{n}
$$

O modelo de Regressão Logística foi formulado pela equação (1.0), na qual $\beta_{0}, \beta_{1}$, $\ldots \beta_{n}$, são os coeficientes das variáveis que explicam a ocorrência de um determinado evento.

Para a construção do modelo de classificação logística a base de dados de 408 alunos foi dividida em 285 alunos (70\%) dos dados para treinamento e 123 alunos (30\%) dos dados para teste, onde a base de treinamento foi composta por observações pertencentes a classe 0 (alunos pertencentes a classe de desempenho insatisfatório) e observações pertencentes à classe 1 (alunos em situação de desempenho insatisfatório). Foi utilizada a técnica de Cross-Validation com 10 folds. Essa técnica possibilitou aumentar a capacidade de generalização do modelo treinado, a partir do conjunto de dados. Esta técnica é amplamente empregada em problemas nos quais o objetivo da modelagem é a predição (Stone, 1974) 
VI Congresso Brasileiro de Informática na Educação (CBIE 2017)

Anais do XXVIII Simpósio Brasileiro de Informática na Educação (SBIE 2017)

\subsubsection{Significância das variáveis no modelo}

No modelo de regressão logística uma das validações que observamos inicialmente foi o teste de hipóteses sobre os coeficientes individualmente. O valor do teste da estatística Wald é utilizado para avaliar a significância de cada coeficiente estimando. Se o coeficiente logístico é estatisticamente significativo $(<0,05)$, podemos interpreta-lo em termos de como o mesmo impacta na previsão de pertinência ao grupo. No quadro 1 são mostradas todas as variáveis e seus respectivos valores de significância.

\begin{tabular}{|c|c|c|c|c|}
\hline \multicolumn{5}{|c|}{ Coefficients: } \\
\hline & Estimate & Std. Error & $z$ value $\operatorname{Pr}(>|z|)$ & \\
\hline (Intercept) & 4.5951467 & 0.0665924 & $54.002<2 e-16$ & $\star \star \star *$ \\
\hline VAR01 & 0.0053145 & 0.0012980 & $4.0944 .23 e-05$ & $\star \star \star$ \\
\hline VAR02 & 0.0971106 & 0.0272446 & 3.5640 .000365 & $\star \star \star$ \\
\hline VAR03 & 0.0140579 & 0.0045620 & -3.0820 .002060 & $\star \star$ \\
\hline VAR0 4 & 0.0025958 & 0.0013660 & $1.900 \quad 0.057381$ & . \\
\hline VAR05 & 0.0024620 & 0.0006952 & 3.5410 .000398 & $\star \star \star \star$ \\
\hline VAR0 6 & 0.2819494 & 0.2156452 & -1.3070 .191054 & \\
\hline VAR0 7 & 0.0582210 & 0.1272019 & $0.458 \quad 0.647164$ & \\
\hline VAR08 & 0.0173138 & 0.0212034 & $0.817 \quad 0.414182$ & \\
\hline VAR09 & 0.0280463 & 0.0183993 & $1.524 \quad 0.127430$ & \\
\hline VAR10 & 0.0669658 & 0.0139889 & $-4.7871 .69 e-06$ & $\star \star \star$ \\
\hline VAR11 & -0.0689171 & 0.0289311 & $-2.382 \quad 0.017214$ & $\star$ \\
\hline VAR12 & 0.0008412 & 0.0055663 & $0.151 \quad 0.879882$ & \\
\hline VAR13 & -0.0393113 & 0.0371802 & -1.0570 .290366 & \\
\hline VAR14 & -1.0816371 & 0.0592768 & $-9.247<2 e-16$ & 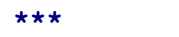 \\
\hline VAR15 & 0.0013450 & 0.0017969 & $0.749 \quad 0.454144$ & \\
\hline VAR16 & 0.0110846 & 0.0018030 & $6.148 \quad 7.86 e-10$ & $\star \star \star$ \\
\hline VAR17 & 0.3991621 & 0.0787712 & $5.0674 .03 e-07$ & $\star \star \star$ \\
\hline VAR18 & -0.1089689 & 0.0137360 & $7.9332 .14 \mathrm{e}-15$ & $\star \star \star \star$ \\
\hline VAR19 & 0.0190252 & 0.0096252 & -1.9770 .048086 & * \\
\hline VAR20 & -0.0165666 & 0.0211643 & 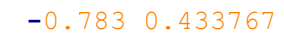 & * \\
\hline VAR21 & -0.1338515 & 0.0721790 & $\begin{array}{lll}-1.854 & 0.063677\end{array}$ & \\
\hline VAR22 & -0.0060239 & 0.0004758 & $-10.661<2 e-16$ & $\star \star \star \star$ \\
\hline VAR23 & 0.0013602 & 0.0007590 & 1.7920 .073119 & . \\
\hline VAR2 4 & 0.0175758 & 0.0061181 & $2.873 \quad 0.004069$ & $\star \star \star$ \\
\hline \multicolumn{5}{|l|}{---} \\
\hline Signif. code & $0 \quad \cdot * \star \star \prime \prime$ & $0.001 * * 1^{\prime}$ & 0.01 '*' 0.05 ' & $.^{\prime} 0.1, '$ \\
\hline
\end{tabular}

O modelo final ficou com 18 variáveis significativas $(p<0,05)$ pertencentes ao modelo final. É possível verificarmos que todas as variáveis do construto Estabelecimento de Metas (var1, var2, var3, var4 e var5) foram significativas para o modelo. Para o construto Estruturação do Ambiente (var6, var7, var8, var9) nenhuma das variáveis foram significativas. Em relação ao construto Estratégia para realização de tarefas tiveram duas variáveis significativas (var10*, var11*, var12, var13). Para o construto Gerenciamento do Tempo (var14*, var15, var16*) tiveram duas variáveis significativas. Os demais construtos Busca por Ajuda e Auto Avaliação obtiveram todas as variáveis significativas.

\subsubsection{Validação do modelo de regressão}

Após a construção do modelo de regressão logística foi realizada uma avaliação do poder de precisão do modelo. A partir da matriz de confusão gerada o modelo conseguiu alcançar um valor de acurácia de 85,3\% de previsão correta e 84,8 de precisão, o que indica uma ótima taxa de previsão, de acordo com os limiares estabelecidos por (FAVEIRO, BELFIORE, et al., 2009).

Além da métrica de acuraria outra métricas utilizada para verificar o poder de previsão de classificação do modelo foi a área sobre a curva ROC. Nesta métrica obtivemos o valor de $92 \%$ de área sob a curva ROC. A figura 3 mostra a imagem que representa a área de classificação. 
VI Congresso Brasileiro de Informática na Educação (CBIE 2017)

Anais do XXVIII Simpósio Brasileiro de Informática na Educação (SBIE 2017)

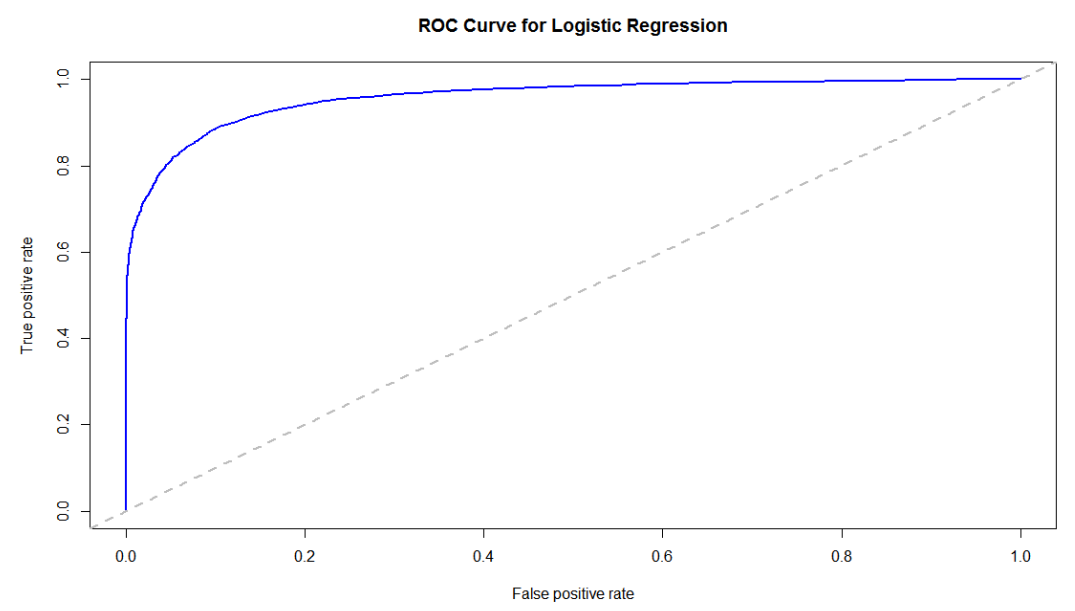

Figura 3. Gráfico da área sob a Curva ROC.

A área da curva é uma porção da área do quadrado unitário (espaço ROC) seus valores variam entre 0 à 1 . Quanto maior a área melhor a performance global do classificador.

Os resultados alcançados com a construção do modelo mostraram-se satisfatórios, de acordo com os limiares propostos por (Hair, Black, Babin, Anderson, \& Tatham, 2009). Estes valores de índices de avaliação nos indicam que o modelo de regressão logística, mostrou-se satisfatório do ponto de vista da acurácia e da métrica da Área sob a curva ROC.

\section{Considerações Finais}

Ainda é pouco expressiva a quantidade de pesquisas sobre a mensuração das características de autorregulação da aprendizagem com foco em ambientes de EAD. A maioria dos instrumentos de coleta disponibilizados na literatura para a mensuração de SRL foram desenvolvidos para ambientes presenciais de ensino.

Esse artigo demonstrou o potencial do questionário OLSQ como instrumento suficiente para coleta de dados que possam a ser utilizados para prever o desempenho acadêmico de alunos de acordo com suas habilidades de autorregulação da aprendizagem.

Foi possível prever o desempenho dos alunos baseado nas suas características e habilidades comportamentais de autorregulação. Os resultados desta pesquisa vem a contribuir com a comunidade científica que busca entender os fenômenos de autorregulação da aprendizagem e sua relação com o desempenho acadêmico de alunos, especificamente em contextos da aprendizagem à distância. A construção do modelo de previsão e seu bom desempenho, a partir das variáveis do questionário possibilitará que outros pesquisadores possam utilizar o instrumento OSLQ em outras instituições, sendo capaz de fornecer indícios de previsibilidade ao professor para tomada de decisão e condução da ação docente.

\section{Agradecimentos}

Esta pesquisa é apoiada pela FACEPE-APQ No. 0525-1.03/14. Os autores também agradecem ao apoio do NEAD/UPE. Alex Sandro Gomes é bolsista DT Nível 2/CNPq, processos no $310466 / 2012-1$ e no 475634/2013-6 e agradecem PRPPG/UFRPE. 
VI Congresso Brasileiro de Informática na Educação (CBIE 2017)

Anais do XXVIII Simpósio Brasileiro de Informática na Educação (SBIE 2017)

\section{Referências}

Agustiani, H., Cahyad, S., \& Musa, M. (2016). Self-efficacy and Self-Regulated Learning as Predictors of Students Academic Performance. The Open Psychology Journal.

Azevedo, R. (2005). Using hypermedia as a metacognitive tool for enhancing student learning? The role of self-regulated learning. Educational Psychologist, 199-209.

Barnard, L., Lan, W. Y., Crooks, S. M., \& Paton, V. O., V. O. (2008). The relationship of epistemological beliefs with self-regulatory skills in the online course environment. Journal of Online and Learning Teaching.

Barnard, L., Lan, W. Y., To, Y. M., Paton, V. O., \& Lai, S. L. (2009). Measuring selfregulation in online and blended learning environments. The Internet and Higher Education.

Beishuizen, J., \& Steffens, K. (2011). A conceptual framework for research on selfregulated learning.

Bolfarine, H., \& Bussab, O. H. (2005). Elementos de amostragem. São Paulo: Edgard Blücher.

Broadbent, J., \& Poon, W. L. (2015). Self-regulated learning strategies \& academic achievement in online higher education learning environments: A systematic review. The Internet and Higher Education, pp. 1-13.

Carson, A. D. (2011). Predicting student success from the LASSI for learning online (LLO). Journal of Educational Computing Research, pp. 399-414.

Chang, M. M. (2010). Effects of self-monitoring on web-based language learner's performance and motivation. CALICO Journal, pp. 298-310.

ChanLin, L. J. (2012). Learning strategies in web-supported collaborative project. Innovations in Education and Teaching International, pp. 319-331.

Cho, M. H., \& Shen, D. (2013). Self-regulation in online learning. Distance Education, pp. 290-301.

Dabbagh, N., \& Kitsantas, A. (2004). Supporting self-regulation in student-centered webbased learning environments. International Journal on E-learning, 40-47.

Dabbagh, N., \& Kitsantas, A. (2005). Using web-based pedagogical tools as scaffolds for self-regulated learning. Instructional Science, 513-540.

Dignath, C., \& Buttner, G. (2008). Components of fostering self-regulated learning among students. A meta-analysis on intervention studies at primary and secondary school level. Metacognition Learning, pp. 231-264.

Garrison, D. R. (2003). Self-directed learning and distance education. Handbook of distance education, 161-168.

Hair, J. F., Black, W. C., Babin, B. J., Anderson, R. E., \& Tatham, R. L. (2009). Análise multivariada de dados. Bookman Editora.

Hartley, K., \& Bendixen, L. D. (2001). Educational research in the Internet age: Examining the role of individual characteristics. Educational Researcher, 22-26. 
VI Congresso Brasileiro de Informática na Educação (CBIE 2017)

Anais do XXVIII Simpósio Brasileiro de Informática na Educação (SBIE 2017)

Hodges, C. B., \& Kim, C. (2010). Email, self-regulation, self-efficacy, and achievement in a college online mathematics course. Journal of Educational Computing Research, pp. 207-223.

Johnson, R. D., Gueutal, H., \& Falbe, C. M. (2009). Technology, trainees, metacognitive activity and e-learning effectiveness. Journal of Managerial Psychology, pp. 545566.

Kinzie , M. B. (1990). Requirements and benefits of effective interactive instruction: Learning control, self-regulation, and continuing motivation. Educational Technology Research and Development, 5-21.

Klingsieck, K. B., Fries, S., Horz, C., \& Hofer, M. (2012). Procrastination in a distance univer- sity setting. Distance Education, pp. 295-310.

Michinov, N., Brunot, S., Le Bohec, O., Juhel, J., \& Delaval, M. (2011). Procrastination, par- ticipation, and performance in online learning environments. Computers \& Education, pp. 243-252.

Pintrich, P. R. (2004). A conceptual framework for assessing motivation and selfregulated learning in college students. Educational Psychology Review, pp. 385407.

Richardson, M., Abraham, C., \& Bond, R. (2012). Psychological correlates of university students' academic performance. A systematic review and meta-analysis. Psychological Bulletin, pp. 353-387.

Schunk, D. H., \& Zimmerman, B. J. (1998). Self-regulated learning: From teaching to self-reflective practice. Guilford Press.

Stone, M. (1974). Cross-validatory choise and assessment of statistical predictions. Journal of the Royal Statistical Society, 111-148.

Van den Boom, G., Paas, F., \& van Merriënboer, J. J. (2007). Effects of elicited reflections combined with tutor or peer feedback on self-regulated learning and learning outcomes. Learning and Instruction, pp. 532-548.

Wang, C. H., Shannon, D., \& Ross, M. (2013). Students' characteristics, self-regulated learning, technology self-efficacy, and course outcomes in online learning. Distance Education, pp. 302-323.

Zimmerman, B. J. (2008). Investigation self-regulation and motivation: historical background, methodological developments, and future prospects. American Educational Research Journal, pp. 166-183. 